\title{
FISIOLOGÍA POSCOSECHA, COMPOSICIÓN QUÍMICA Y CAPACIDAD ANTIOXIDANTE DE FRUTAS DE PEJIBAYE (Bactris gasipaes KUNTH) CV. TUIRA DARIÉN COSECHADAS A TRES DIFERENTES EDADES ${ }^{1}$
}

\author{
Mauricio Serrano*, Gerardina Umaña ${ }^{2 / *}$, Marco V. Sáenz*
}

\begin{abstract}
Palabras clave: Bactris gasipaes, pejibaye, calidad, respiración, poscosecha, fruto no climatérico, capacidad antioxidante.

Keywords: Bactris gasipaes, peach palm, quality, respiration, postharvest, non-climacteric fruit, antioxidant
\end{abstract} capacity.

Recibido: 06/07/11

\section{RESUMEN}

Se determinaron variables de calidad poscosecha, composición química y capacidad antioxidante de frutas de pejibaye cv. Tuira-Darién, cosechados con una edad de 97, 106 y 120 días desde floración. Con base en su patrón respiratorio, el pejibaye pareciera pertenecer al grupo de los frutos no climatéricos, con una tasa respiratoria alta $\left(30-45 \mathrm{mg} \cdot \mathrm{kg}^{-1} \cdot \mathrm{h}^{-1}\right.$ de $\mathrm{CO}_{2}$ al inicio del almacenamiento; en etapas más avanzadas del almacenamiento alcanzó $80 \mathrm{mg} \cdot \mathrm{kg}^{-1} \cdot \mathrm{h}^{-1} \mathrm{de} \mathrm{CO}_{2}$ ), lo que le confiere una vida útil poscosecha corta. En los frutos recién cosechados, los valores de firmeza de la pulpa, el color de la pulpa, el color externo, el contenido de sólidos solubles, el contenido de grasa y la capacidad antioxidante se vieron influenciados por la edad de cosecha. No se encontró efecto de la edad de cosecha en el contenido de almidones y carotenoides. Los valores de respiración, índice de color externo y contenido de sólidos solubles aumentaron durante el almacenamiento poscosecha, mientras que la firmeza de la pulpa mostró una disminución. Se encontraron bajos contenidos de acidez titulable, sin variaciones significativas durante el periodo de almacenamiento. Además, mediante el método ORAC en base húmeda, se determinó que el

1 Este trabajo es parte de la tesis M.Sc. del primer autor. Programa de Estudios de Posgrado en Ciencias Agrícolas y Recursos Naturales, Universidad de Costa Rica.
Aceptado: 18/10/11

\begin{abstract}
Postharvest physiology, chemical composition and antioxidant capacity of peach palm fruit (Bactris gasipaes Kunth) cv. Tuira Darién, harvested at three different ages. Postharvest quality, chemical composition and antioxidant activity were evaluated on peach palm fruit harvested at 97, 106 and 120 days after blooming. Based on the respiration pattern, peach palm appears to be a non-climacteric fruit, which has a high respiration rate $\left(30-45 \mathrm{mg} \cdot \mathrm{kg}^{-1} \cdot \mathrm{h}^{-1} \mathrm{CO}_{2}\right.$ a the beginning of storage $\left(12^{\circ} \mathrm{C}\right)$ and $80 \mathrm{mg} \cdot \mathrm{g}^{-1} \cdot \mathrm{h}^{-1} \mathrm{CO}_{2}$ at the end of storage, which implies a short pos-harvest life. External color, pulp color, pulp firmness, soluble solids content, fat content and antioxidant capacity were influenced by harvest age in recently-harvested fruit. There was no effect of harvest age on the content of starch and carotenoids. External color index, respiration values and soluble solids content increased during postharvest storage, while pulp firmness declined. Low acidity contents were found, without significant changes during storage. Using the ORAC method it was determined that the fruit has a high antioxidant capacity $16.18 \mu \mathrm{mol}$ $\mathrm{TE} / \mathrm{g}$ on a humid basis, which is higher than reported in other fruits (mango $10.02 \mu \mathrm{mol} \mathrm{TE} / \mathrm{g}$, pineapple $7.93 \mu \mathrm{mol} \mathrm{TE} / \mathrm{g}$, tomato $4.60 \mu \mathrm{mol}$
\end{abstract}

2 Autor para correspondencia. Correo electrónico: gerardina.umana@ucr.ac.cr

* Laboratorio de Tecnología Poscosecha, Centro de Investigaciones Agronómicas, Universidad de Costa Rica. San José, Costa Rica. 
pejibaye tiene una alta capacidad antioxidante (16,18 $\mu \mathrm{mol}$ ET (equivalentes Trolox)/g), superior a la informada para frutas como mango $(10,02$ $\mu \mathrm{mol} \mathrm{ET/g})$, piña $(7,93 \mu \mathrm{mol} \mathrm{ET} / \mathrm{g})$, tomate $(4,60$ $\mu \mathrm{mol} \mathrm{ET/g})$. Los frutos cosechados con la menor edad (97 días), presentaron los mayores valores de capacidad antioxidante tanto en la determinación ORAC en base seca $(58,08 \mu \mathrm{mol} \mathrm{ET/g})$ como en la base húmeda $(16,18 \mu \mathrm{mol} \mathrm{ET/g})$.

\section{INTRODUCCIÓN}

El pejibaye cultivado (Bactris gasipaes Kunth) es el resultado de la hibridación, selección natural y domesticación de varias especies silvestres de palmas nativas del trópico húmedo americano. Su distribución abarca desde Nicaragua hasta Bolivia. Tuvo gran importancia y desarrollo durante la época precolombina, cuando posiblemente se constituyó en el principal cultivo para varias tribus de Centro y Suramérica (MoraUrpí 1983).

Actualmente el pejibaye es de gran aceptación en nuestro país, lo que facilita su promoción y permite sacar provecho de su valor nutritivo. Algunos de los nutrientes más relevantes en este fruto son las grasas, almidones, minerales, fibras de origen vegetal y carotenoides (Fernández 1988). También es una fuente importante de niacina, riboflavina, tiamina, hierro y retinol (Gómez 1990, Mora-Urpí et al. 1997). En este fruto se han encontrado altos contenidos de carotenoides, los cuales están involucrados en el fortalecimiento del sistema inmunológico y disminución del riesgo de enfermedades como cáncer, enfermedades cardiovasculares y artritis entre otros. Estos efectos biológicos se atribuyen a la capacidad antioxidante que poseen, a través de la cual desactivan radicales libres y oxígenos reactivos en el organismo humano (RodriguezAmaya 1997).

En los últimos años investigadores y consumidores han mostrado interés hacia el consumo
TE/g). The youngest fruit (97 days) exhibited the highest antioxidant capacity on both dry basis $(58,08 \mu \mathrm{mol} \mathrm{TE} / \mathrm{g})$ and humid basis $(16,18 \mu \mathrm{mol}$ $\mathrm{TE} / \mathrm{g})$.

de frutas exóticas y con alta capacidad antioxidante, lo cual ha impulsado la búsqueda de productos tropicales promisorios y poco investigados como el pejibaye. Sin embargo, la comercialización de esta fruta se dificulta ya que bajo condiciones naturales la vida poscosecha del pejibaye es limitada, lo que provoca grandes pérdidas en poscosecha. En tan solo 4 días a temperatura ambiente, hasta un $70 \%$ de la fruta cruda de pejibaye puede sufrir algún grado de deterioro por enfermedades (Sáenz et al. 1992).

Adicionalmente existe un desconocimiento de la fisiología poscosecha de la fruta del pejibaye y de las alternativas que podrían ser utilizadas para extender la vida poscosecha. No se tiene información sobre el comportamiento respiratorio, y sobre variables de calidad poscosecha como el color interno, color externo, cambios en firmeza, contenido de sólidos solubles y acidez.

En relación con la composición química y valor nutritivo del pejibaye, existen resultados muy diversos que se explican tanto por los métodos de análisis, como por la variabilidad genética existente entre los materiales evaluados. Adicionalmente, los resultados de análisis químicos no se han relacionado con índices de cosecha (edad del racimo), para así precisar los cambios que ocurren durante el desarrollo del racimo.

Por lo anterior, el objetivo de este trabajo fue determinar el comportamiento fisiológico y la composición química, la capacidad antioxidante de la fruta de pejibaye con 3 diferentes edades de cosecha. 


\section{MATERIALES Y MÉTODOS}

El experimento se realizó durante junio a octubre del 2006, con fruta de pejibaye de la cv. Tuira-Darién (Hernández et al. 2008, Mora et al. 1999), procedente del banco de germoplasma de pejibaye perteneciente a la Finca Experimental "Los Diamantes", ubicada en la localidad de Guápiles, cantón de Pococí, provincia de Limón, a $10^{\circ}$ 13' latitud norte y $86^{\circ} 46^{\prime}$ longitud oeste, a una altitud de $249 \mathrm{msnm}$. La precipitación promedio anual es de $4500 \mathrm{~mm}$ y la temperatura promedio es $25,9^{\circ} \mathrm{C}$.

Se seleccionaron 24 plantas, en las que se identificaron inflorescencias recién polinizadas las que fueron marcadas para luego cosechar los racimos una vez transcurridos 97, 106 y 120 días desde el momento de la floración.

Los racimos se transportaron en cajas plásticas abiertas dentro de un vehículo, protegidos del sol, con ventilación y a temperatura ambiente hasta el Laboratorio de Tecnología Poscosecha del Centro de Investigaciones Agronómicas de la Universidad de Costa Rica. Entre el momento de la cosecha, transporte y la aplicación de los tratamientos transcurrió un periodo aproximado de 6 horas.

Los frutos se separaron del racimo con el cáliz adherido al fruto; se seleccionaron, lavaron y desinfectaron en una solución de hipoclorito de sodio a una concentración de $60 \mathrm{mg} \cdot \mathrm{l}^{-1}$ durante un min. Se descartaron aquellos que presentaban pudriciones, daños mecánicos o mala apariencia.

Después del proceso de lavado y selección, los frutos se colocaron en cajas plásticas abiertas con ranuras laterales. Cada caja contenía aproximadamente $2 \mathrm{~kg}$ de frutos provenientes de un único racimo. Se almacenaron en una cámara a $16^{\circ} \mathrm{C}$ y humedad relativa entre $80 \%$ y $90 \%$ determinada con un higrómetro digital Delta Track modelo 13307.

El diseño experimental utilizado fue irrestricto al azar con 3 tratamientos (97, 106 y 120 días floración-cosecha) y 8 repeticiones por tratamiento para un total de 24 unidades experimentales. Cada unidad experimental correspondió a una caja.
Al momento de la cosecha se evaluó el contenido de grasa, almidón total, carotenoides totales y capacidad antioxidante total. Además, cada 5 días se evaluaron los cambios en el porcentaje de sólidos solubles, acidez titulable, color interno (pulpa), color externo (epicarpo) y firmeza de pulpa. La pérdida de peso y la tasa respiratoria se evaluó cada 2 o 3 días durante la duración del almacenamiento.

Para las variables firmeza, color interno, sólidos solubles, acidez y tasa respiratoria se realizaron 24 mediciones por tratamiento, mientras que para el color externo se evaluaron 20 frutos por unidad experimental, para un total de 160 observaciones por tratamiento.

El color externo e interno se evaluó sobre 3 puntos equidistantes en el plano ecuatorial del fruto con un colorímetro Minolta ${ }^{\circledR}$ CR-200 mediante la escala CIE L*a*b*. En esta escala la coordenada "L" es una medida de la claridad (blanco-negro y varía desde el punto de ausencia de reflejo, $\mathrm{L}=0$, hasta el punto de reflejo difuso perfecto, $\mathrm{L}=100$ ). La escala "a" varía desde los valores negativos para el color verde, hasta los positivos para el rojo. La escala "b" varía desde los valores negativos para el color azul, hasta los positivos para el amarillo. Con los valores obtenidos se calculó un índice de color (IC) mediante la fórmula $\mathrm{IC}=1000 * \mathrm{a} / \mathrm{L} * \mathrm{~b}$.

Para la determinación del contenido de sólidos solubles (grados Brix) y la acidez titulable se homogeneizó la pulpa durante un min en licuadora, con una dilución de 3 veces la masa de la muestra y se filtró con papel filtro cualitativo. Cada fruto constituyó una muestra diferente. El jugo filtrado se evaluó con un refractómetro digital Atago Pal-1. Para determinar la acidez titulable se utilizó un titulador automático Orion 960SC. Se calculó el contenido de sólidos solubles y acidez titulable presente en la pulpa mediante el factor de dilución utilizado en la solución homogeneizada.

La firmeza expresada en Newton (N) se evaluó en la pulpa sobre caras opuestas de la fruta con un penetrómetro Chatillon con punta cónica de $6 \mathrm{~mm}$ de diámetro. Antes de realizar la evaluación, con un cuchillo se eliminó el epicarpo del 
fruto de manera superficial, y a su vez se eliminó la mínima cantidad posible de pulpa durante el corte.

La tasa respiratoria se analizó mediante la utilización de un sistema cerrado, el cual consiste en la colocación de una cantidad de frutos de peso conocido dentro de un recipiente de volumen conocido cerrado herméticamente durante un periodo de $1 \mathrm{~h}$. Se evaluó el \% de $\mathrm{CO}_{2}$ y el $\%$ de $\mathrm{O}_{2}$ dentro del sistema con un analizador de gases Illinois Instruments 6600. Se calculó la tasa respiratoria en términos de producción de dióxido de carbono (mg.kg-1 $\mathrm{h}^{-1}$ de $\mathrm{CO}_{2}$ ), y con el consumo de oxígeno $\left(\mathrm{mg}_{\mathrm{kg}} \mathrm{kg}^{-1} \cdot \mathrm{h}^{-1}\right.$ de $\mathrm{O}_{2}$ ) se calculó el cociente respiratorio $\left(\mathrm{CO}_{2} / \mathrm{O}_{2}\right)$.

La determinación del contenido de carotenoides se realizó mediante el método AQCITAM035 (CITA 2007), modificado de Britton et al. (1995), Mencaca y Murillo (2006), y RodríguezAmaya (1999). La capacidad antioxidante de las muestras se determinó mediante el método ORAC (oxygen radical absorbance capacity) descrito por $\mathrm{Ou}$ et al. (2001). El almidón total se calculó mediante la sumatoria entre el almidón potencialmente disponible y el almidón resistente. Para determinar el almidón disponible se utilizó la metodología multienzimática descrita por Holm et al. (1986), mientras que para determinar el almidón resistente se utilizó el método descrito por SauraCalixto et al. (1993). Para determinar el contenido de grasa se realizó hidrólisis ácida de la muestra y determinación del extracto etéreo mediante el sistema Soxhlet descrito por AOAC (1990).

Con los datos obtenidos en cada variable se realizó un análisis de varianza y se graficaron los promedios. A los resultados de análisis químico se les realizó un análisis de varianza y una separación de medias DMS.

\section{RESULTADOS Y DISCUSIÓN}

La Figura 1 muestra los resultados de respiración para frutos de pejibaye con 3 edades de cosecha: 97, 106 y 120 días floración-cosecha; para cada edad de cosecha se utilizaron 8 racimos provenientes de diferentes plantas. Durante los primeros 6 días de almacenamiento los valores de respiración se mantuvieron estables. A partir de los 8 días los 3 tratamientos presentaron un incremento en su tasa respiratoria que coincidió con la degradación progresiva que se observó en la fruta.

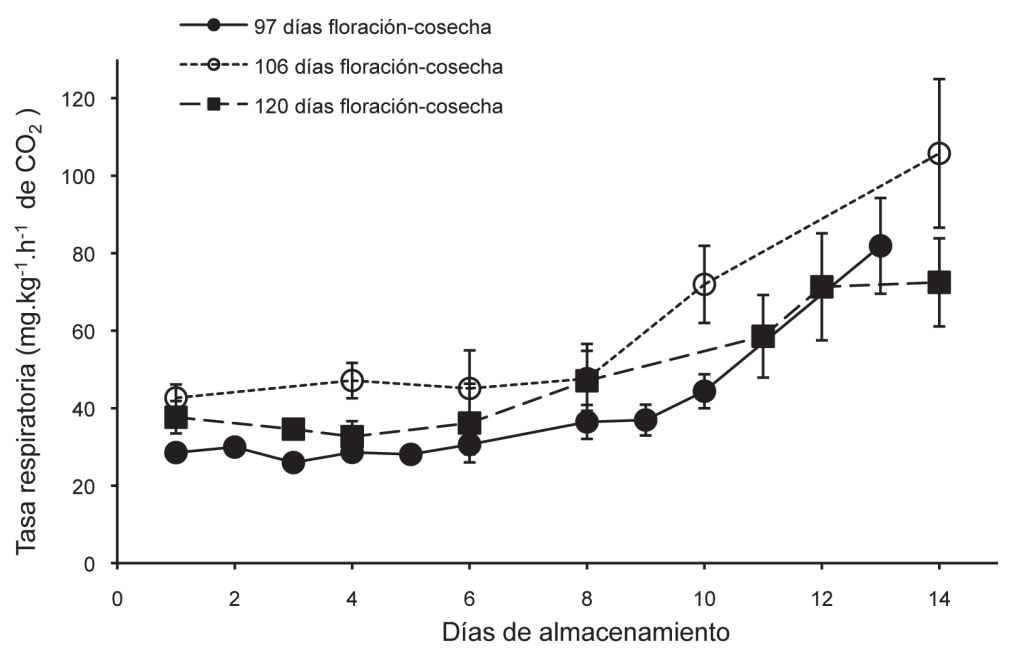

Fig. 1. Tasa respiratoria promedio $\left(\mathrm{mg} \cdot \mathrm{kg}^{-1} \cdot \mathrm{h}^{-1} \mathrm{de} \mathrm{CO}_{2}\right) \pm$ intervalo de confianza $95 \%$ de frutos de pejibaye (Bactris gasipaes Kunth) cv. Tuira-Darién con 3 distintas edades de cosecha durante su almacenamiento a $16^{\circ} \mathrm{C}$. 
La magnitud de la tasa respiratoria en pejibaye puede considerarse alta en comparación con varios frutos no climatéricos, en los cuales se ha determinado valores máximos cercanos a $30 \mathrm{mg} \cdot \mathrm{kg}^{-1} \cdot \mathrm{h}^{-1}$ de $\mathrm{CO}_{2}$ en uva, $25 \mathrm{mg} \cdot \mathrm{kg}^{-1} \cdot \mathrm{h}^{-1}$ de $\mathrm{CO}_{2}$ en cereza, $20 \mathrm{mg} \cdot \mathrm{kg}^{-1} \cdot \mathrm{h}^{-1}$ de $\mathrm{CO}_{2}$ en piña, $10 \mathrm{mg} \cdot \mathrm{kg}^{-1} \cdot \mathrm{h}^{-1}$ de $\mathrm{CO}_{2}$ en limón (Seymour et al. 1993) y en órganos subterráneos como zanahoria (19 mg.kg-1 $\mathrm{h}^{-1}$ de $\mathrm{CO}_{2}$ ), papa (20 mg.kg ${ }^{-1} \cdot \mathrm{h}^{-1} \mathrm{de}$ $\mathrm{CO}_{2}$ ), cebolla (7 mg. $\mathrm{kg}^{-1} \cdot \mathrm{h}^{-1}$ de $\mathrm{CO}_{2}$ ) (Kays 1997), mientras que para pejibaye se obtuvieron valores de respiración entre 30 y $45 \mathrm{mg} \cdot \mathrm{kg}^{-1} \cdot \mathrm{h}^{-1}$ de $\mathrm{CO}_{2}$ al inicio del almacenamiento, y en etapas más avanzadas del almacenamiento se alcanzó con facilidad $80 \mathrm{mg} \cdot \mathrm{kg}^{-1} \cdot \mathrm{h}^{-1} \mathrm{de} \mathrm{CO}_{2}$. Esto explica en cierta medida la corta duración de la vida útil del pejibaye ya que en frutos no climatéricos existe una correlación entre tasas respiratorias elevadas y una corta vida útil poscosecha (Seymour et al. 1993).

$\mathrm{Al}$ inicio (día 1) del almacenamiento (Figura 1) se encontraron diferencias significativas entre los frutos cosechados a los 106 y 120 días, los cuales mostraron valores de respiración más altos, en comparación con los frutos cosechados a los 97 días del periodo floración cosecha $(\mathrm{p}<0,0001)$. En muchos frutos no climatéricos por lo general existe una correlación entre tasas respiratorias elevadas y una corta vida útil poscosecha (Seymour et al. 1993), por lo cual era de esperar una mayor tasa respiratoria para los frutos de pejibaye cosechados con mayor cantidad de días floración-cosecha, dado que estos por lo general tienen una vida útil poscosecha más corta (Mora-Urpí 1983).

Se realizó una regresión a partir del día 8 de almacenamiento (dado que los días, la fruta se encontraba en un periodo de respiración sin mayor variación), esto como herramienta para diferenciar entre los patrones de respiración de un fruto no climatérico y un fruto climatérico. Los valores de $\mathrm{R}^{2}$ obtenidos (Figura 2), muestran que existe un buen ajuste lineal de los datos, lo que es un indicador de un aumento lineal de la respiración durante el almacenamiento.

Con base en el patrón respiratorio que un fruto muestra durante el almacenamiento, éste puede clasificarse como climatérico o no climatérico. Los frutos climatéricos experimentan un pico característico en su actividad respiratoria durante su maduración, el cual puede corresponder o anteceder a su estado óptimo para ser consumido, usualmente los incrementos respiratorios siguen un patrón exponencial hasta alcanzar el máximo y posteriormente se produce una caída en la

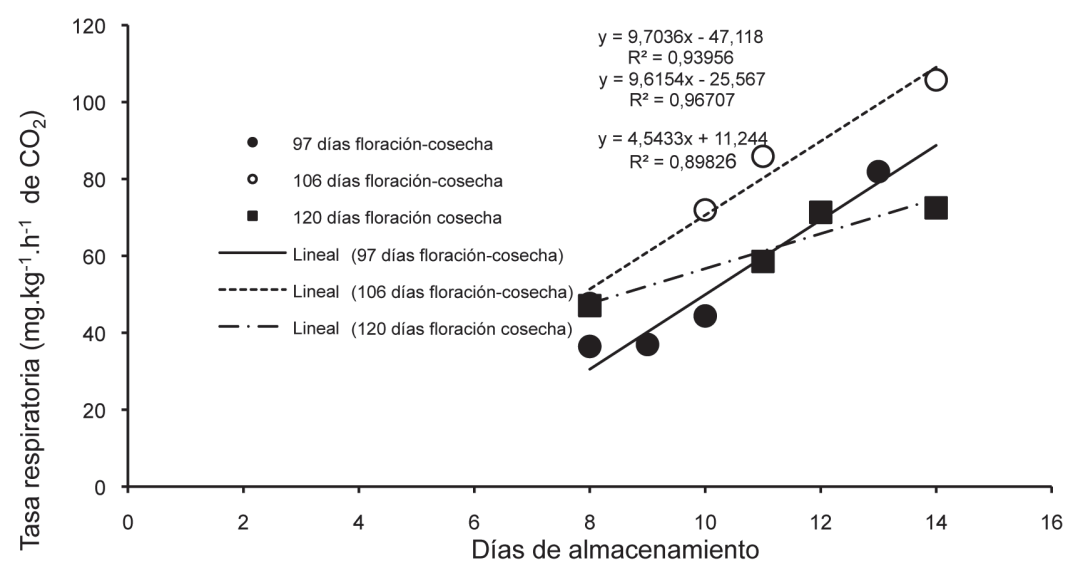

Fig. 2. Tasa respiratoria promedio $\left(\mathrm{mg} \cdot \mathrm{kg}^{-1} \cdot \mathrm{h}^{-1} \mathrm{de} \mathrm{CO}_{2}\right.$ ) de frutos de pejibaye (Bactris gasipaes Kunth) cv. Tuira-Darién con 3 distintas edades de cosecha durante su almacenamiento a $16^{\circ} \mathrm{C}$. Se presenta la ecuación de regresión lineal y $\mathrm{R}^{2}$ para los datos registrados entre los 8 y 14 días de almacenamiento. 
actividad. En contraste, los frutos no climatéricos pueden experimentar un incremento en su actividad respiratoria durante su almacenamiento, con un patrón de tipo lineal sin evidencias de un pico o punto máximo definido (Goldschmidt 1997).

Sin embargo, existen algunos frutos no climatéricos como fresa, carambola y rambután, que pueden mostrar un incremento gradual en su tasa respiratoria durante el tiempo de almacenamiento. En estas 3 frutas con la senescencia ocurre un aumento en la tasa respiratoria, así como un aumento en la susceptibilidad a pudriciones causadas por hongos (O’Hare 1997a, O'Hare 1997b, Bower et al. 2003). De manera similar a la carambola, el patrón de respiración del fruto de pejibaye (Figura 1) correspondería al de un fruto no climatérico, en el cual se produce un aumento en la respiración con el transcurrir del tiempo de almacenamiento, especialmente a partir de los 8 días, cuando el fruto se vuelve más susceptible a la acción de microorganismos que aceleran su deterioro. Es la primera investigación donde se clasifica al pejibaye como no climatérico, sin embargo, queda pendiente para terminar de realizar esta clasificación, el estudio de producción de etileno en poscosecha.

Los valores de cociente respiratorio obtenidos en la Figura 3 mostraron valores que según Kays (1997) indicarían que el fruto de pejibaye utiliza de manera preferente carbohidratos como

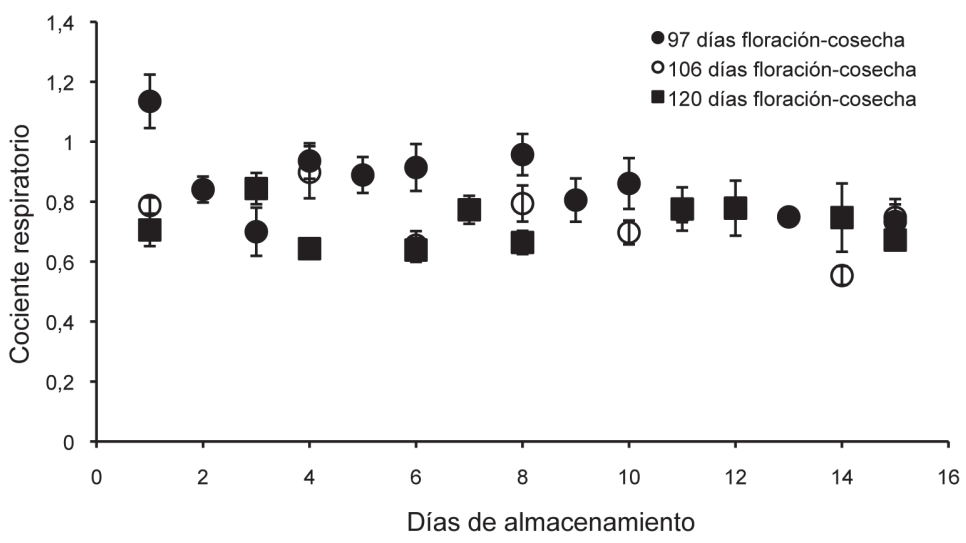

Fig. 3. Cociente respiratorio promedio $\left(\mathrm{mg} \cdot \mathrm{kg}^{-1} \cdot \mathrm{h}^{-1}\right.$ de $\left.\mathrm{CO}_{2} / \mathrm{mg} \cdot \mathrm{kg}^{-1} \cdot \mathrm{h}^{-1} \mathrm{de} \mathrm{O}_{2}\right) \pm$ intervalo de confianza $95 \%$ de frutos de pejibaye (Bactris gasipaes Kunth) cv.Tuira-Darién con 3 distintas edades de cosecha durante su almacenamiento a $16^{\circ} \mathrm{C}$.

sustrato para la respiración, aunque la utilización de lípidos como sustrato en una menor medida también podría estar presente. El cociente respiratorio es un coeficiente que expresa la cantidad de moléculas de dióxido de carbono liberadas en relación con la cantidad de moléculas de oxígeno absorbido, y se utiliza para determinar cuál sustrato es utilizado principalmente para producir energía durante la respiración. Estos sustratos por lo general son carbohidratos, lípidos o ácidos orgánicos (Kays 1997).
En términos generales cocientes respiratorios entre 0,7 y 1,3 corresponden a respiración aeróbica (Salveit 2004, citado por Schouten et al. 2009), mientras que cocientes respiratorios muy por debajo de 1 se obtienen cuando el fruto utiliza principalmente lípidos como sustrato para la respiración (Kays 1997).

La firmeza de la pulpa en la Figura 4 fue menor para los índices de cosecha más altos $(\mathrm{p}<0,0001)$. La firmeza disminuyó hacia los últimos días de almacenamiento para los frutos 


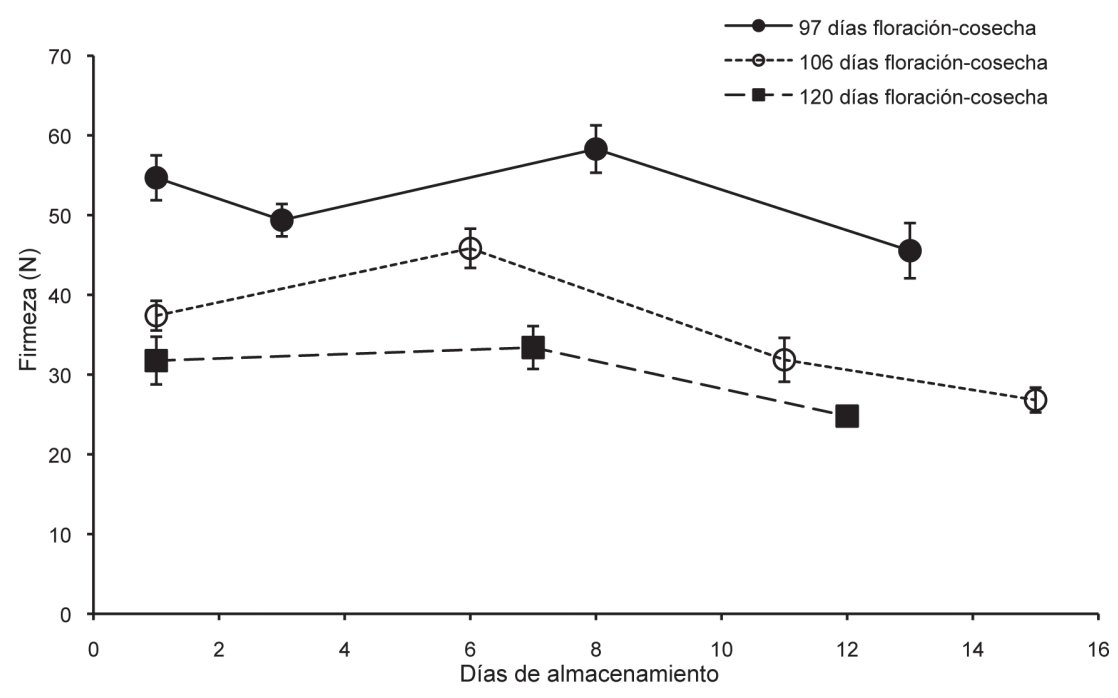

Fig. 4. Firmeza (Newton) \pm intervalo de confianza 95\% de la pulpa de frutos de pejibaye (Bactris gasipaes Kunth) cv. TuiraDarién con 3 distintas edades de cosecha durante su almacenamiento a $16^{\circ} \mathrm{C}$.

cosechados con $97(\mathrm{p}=0,0001), 106(\mathrm{p}<0,0001)$ y 120 días de periodo floración-cosecha ( $\mathrm{p}=0,0001)$. En el caso de la fruta fresca de pejibaye, es deseable que la pulpa sea firme, ya que el ablandamiento está relacionado con el deterioro durante su vida poscosecha y con una menor aceptación por parte de los consumidores (Garaico y Espinoza 1993). De ahí que los frutos con índices de cosecha altos fueron más perecederos y presentaron más dificultades para su almacenamiento y manipulación en fresco.

La pérdida de firmeza se produce a través de 3 mecanismos: pérdida de la turgencia celular, degradación de almidones y ruptura de las paredes celulares que es la de mayor importancia en la mayoría de frutas (Seymour et al. 1993). Según la fruta, el efecto de la pérdida de firmeza puede ser detrimental (como en el caso del pejibaye), mientras que en otras la pérdida de firmeza es un componente necesario para alcanzar la calidad óptima para el consumo (Kays 1997).

La Figura 5 muestra los valores de pérdida de peso. A los 6 días de almacenamiento se obtuvieron valores promedio de pérdida de peso entre $2,8 \%$ y $4,2 \%$, mientras que posterior a los
12 días se obtuvieron valores que superaron el $7 \%$ de pérdida de peso, los cuales muestran que el pejibaye es un fruto que experimenta una importante pérdida de peso durante el almacenamiento, lo cual podría afectar severamente su calidad y apariencia si se almacenara bajo condiciones adversas. La pérdida de agua por transpiración es una de las causas más importantes del deterioro durante el almacenamiento, ya que la mayoría de frutas y legumbres contienen entre el $80 \%$ y $90 \%$ de agua por peso, parte del cual se pierde por transpiración. Para la mayoría de frutas y vegetales se considera que pérdidas de peso entre $3 \%$ y $6 \%$ son suficientes para producir un marcado desmejoramiento de la calidad (Hardenburg et al. 1998).

En la Figura 6 se presentan los valores de sólidos solubles (grados brix). Se encontraron diferencias significativas $(p=0,0029)$ entre los frutos cosechados con 97 días y los frutos cosechados con 106 y 120 días de periodo floración cosecha al inicio (día 1) del almacenamiento, lo cual demuestra que la edad de cosecha del racimo influye en el contenido de sólidos solubles en el fruto. Aunque pequeño en su magnitud, todos 


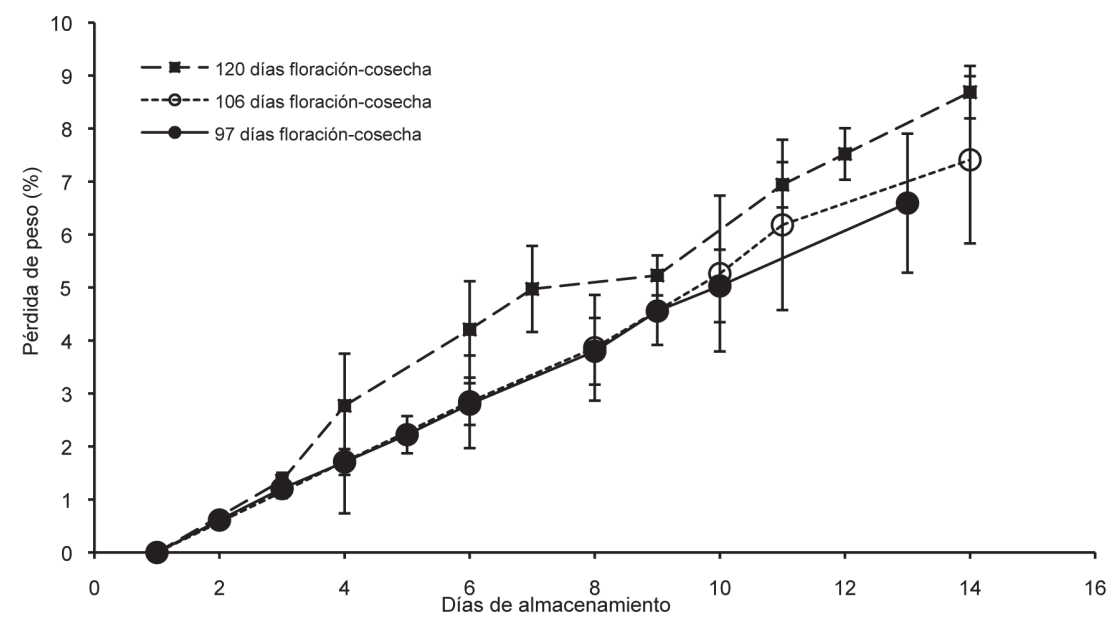

Fig. 5. Pérdida de peso acumulada (\%) \pm intervalo de confianza $95 \%$ de frutos de pejibaye (Bactris gasipaes Kunth) cv. TuiraDarién con 3 distintas edades de cosecha durante su almacenamiento a $16^{\circ} \mathrm{C}$.

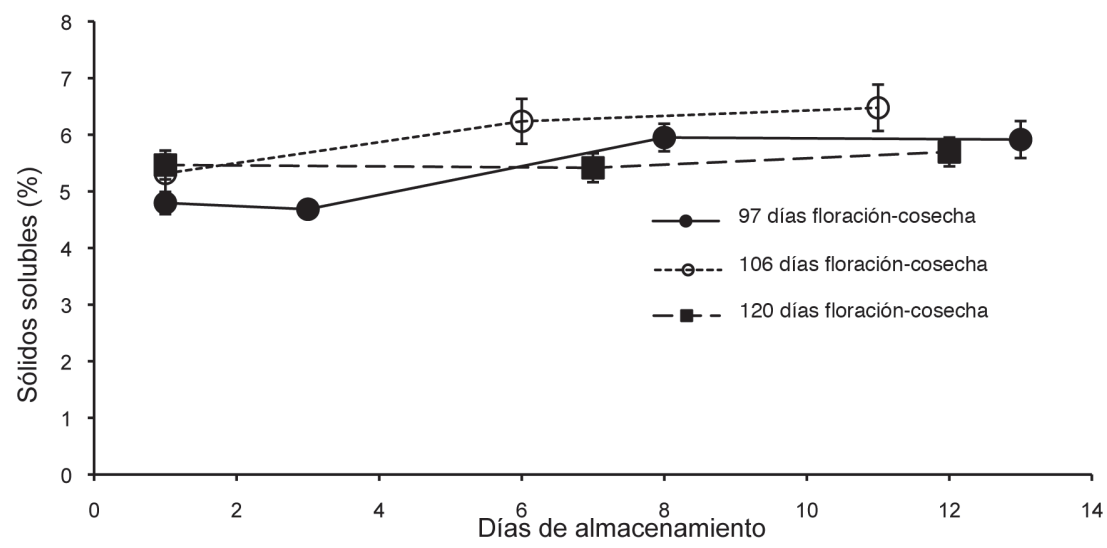

Fig. 6. Contenido de sólidos solubles (\%)土intervalo de confianza 95\% de la pulpa homogeneizada de frutos de pejibaye (Bactris gasipaes kunth) cv. Tuira-Darién con 3 distintas edades de cosecha durante su almacenamiento a $16^{\circ} \mathrm{C}$.

los tratamientos experimentaron un incremento significativo $(p<0,0001)$ en el contenido de sólidos solubles durante el periodo de almacenamiento a $16^{\circ} \mathrm{C}$, lo cual corresponde con los resultados obtenidos por Chaves (2005) quien encontró un ligero aumento en el contenido de sólidos solubles durante un periodo de almacenamiento a $12^{\circ} \mathrm{C}$. Este comportamiento de leve incremento en sólidos solubles es característico de frutos no climatéricos, en comparación a los frutos climatéricos, los cuales posterior a la cosecha experimentan un incremento muy evidente en su contenido de sólidos solubles, debido a la conversión de almidones en azúcares (Giovannoni 2001).

En la acidez titulable no se encontraron diferencias significativas entre tratamientos 
$(\mathrm{p}=0,6576)$. Los frutos evaluados mostraron valores de acidez titulable entre $0,10 \%$ y $0,15 \%$, lo que indica que los ácidos no experimentan cambios relevantes en la fisiología poscosecha del pejibaye, y que además, están presentes en el fruto en una baja cantidad.

Con respecto al índice de color externo (Figura 7), se encontraron diferencias entre tratamientos $(\mathrm{p}<0,0001)$. Los valores mayores se obtuvieron con el tratamiento de 120 días floración-cosecha, debido a una mayor presencia de color rojo en la cáscara (índice con valores positivos) que puede ser atribuido a un mayor contenido de carotenoides, los cuales son abundantes en el fruto de pejibaye y aún más en aquellos que presentan cáscara roja (Johannessen 1967). Por el contrario, el índice fue menor con el tratamiento de 97 días floración-cosecha, debido a una mayor presencia de color verde en la cáscara (índice con valores negativos). Se encontraron también

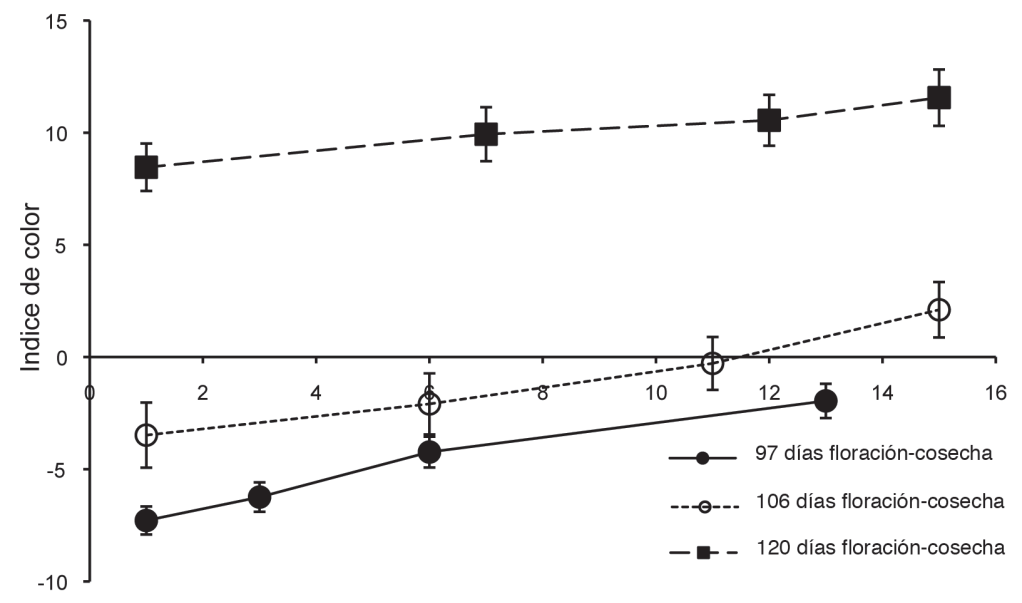

Días de almacenamiento

Fig. 7. Índice de color externo \pm intervalo de confianza $95 \%$ de la cascara de frutos de pejibaye (Bactris gasipaes kunth) cv. Tuira-Darién con 3 distintas edades de cosecha durante su almacenamiento.

diferencias en el índice de color durante el tiempo transcurrido en almacenamiento $(\mathrm{p}<0,0001)$. Este índice aumenta conforme al tiempo de almacenamiento, y concuerda con lo observado por MoraUrpí (1983) quien encontró que el fruto puede ser cosechado verde, y al ser almacenado bajo buenas condiciones de ventilación tiene la capacidad de cambiar el color en su cáscara. Sin embargo, lo que principalmente permite que el índice de color aumente durante el almacenamiento es la degradación de la clorofila, ya que este es un proceso característico de la senescencia y maduración de frutos, en el cual se desenmascaran pigmentos formados con anterioridad durante el desarrollo del fruto (Kays 1997, Matile et al. 1999).

De manera similar al color externo, el color de la pulpa mostró valores más altos a mayores índice de cosecha $(\mathrm{p}=0,0463)$. Pero el valor de este índice no aumenta con el tiempo de almacenamiento en ninguno de los 3 índices de cosecha $(p>0,05)$, lo que indica que en la pulpa del pejibaye no ocurre una producción significativa de pigmentos carotenoides una vez cosechado el fruto.

El Cuadro 1 muestra los contenidos de grasa, almidón total, carotenoides totales y 
Cuadro 1. Promedio \pm desviación estándar de contenido de grasa, almidón total, carotenoides totales y capacidad antioxidante (ORAC) de la pulpa cruda de pejibaye cosechado con 3 edades floración-cosecha. $\mathrm{n}=8$ racimos.

\begin{tabular}{cccccc}
\hline $\begin{array}{c}\text { Edad de cosecha del } \\
\text { racimo }\end{array}$ & $\begin{array}{c}\text { Grasas } \\
(\%)\end{array}$ & Almidón total $(\%)$ & Carotenoides $(\mu \mathrm{g} / \mathrm{g})$ & $\begin{array}{c}\text { ORAC B.S. } \\
(\mu \mathrm{mol} \mathrm{ET} / \mathrm{g})\end{array}$ & $\begin{array}{c}\text { ORAC B.H. } \\
(\mu \mathrm{mol} \mathrm{ET} / \mathrm{g})\end{array}$ \\
\hline 97 días & $7,8 \pm 2,1 \mathrm{a}$ & $64,2 \pm 11,9$ & $129,82 \pm 56,49$ & $58,08 \pm 17,36 \mathrm{~b}$ & $16,18 \pm 5,24$ \\
106 días & $12,0 \pm 4,0 \mathrm{~b}$ & $69,1 \pm 6,5$ & $104,17 \pm 53,63$ & $46,82 \pm 14,54 \mathrm{ab}$ & $15,02 \pm 4,84$ \\
120 días & $12,5 \pm 3,3 \mathrm{~b}$ & $70,7 \pm 3,6$ & $143,68 \pm 81,39$ & $37,82 \pm 11,14 \mathrm{a}$ & $14,72 \pm 4,07$ \\
$\mathrm{P}>\mathrm{F}$ & 0,0935 & 0,3683 & 0,5832 & 0,0997 & 0,0997 \\
\hline
\end{tabular}

Medias dentro de la misma columna con distinta letra difieren significativamente $(\mathrm{p}<0,1)$ según prueba DMS.

Datos calculados en base seca para grasas, almidón total y carotenoides. Los resultados de ORAC se presentan en base seca (BS) y base húmeda $(\mathrm{BH})$.

capacidad antioxidante para las distintas edades de cosecha. Estos en conjunto con otros compuestos y minerales hacen del pejibaye una fruta con un gran valor nutricional (Mora-Urpí et al. 1997), superior por la cantidad y balance de diferentes componentes a cualquier otro producto de los trópicos (León 1987). Adicionalmente, los valores de capacidad antioxidante obtenidos resultaron superiores a los reportados en base húmeda para frutas como mango $(10,02 \mu \mathrm{mol} \mathrm{ET} / \mathrm{g})$, piña $(7,93$ $\mu \mathrm{mol} \mathrm{ET/g})$, banano $(8,79 \mu \mathrm{mol} \mathrm{ET} / \mathrm{g})$, melón $(3,12 \mu \mathrm{mol} \mathrm{ET} / \mathrm{g})$, tomate $(4,60 \mu \mathrm{mol} \mathrm{ET} / \mathrm{g})$, kiwi $(9,18 \mu \mathrm{mol} \mathrm{ET} / \mathrm{g})$ y uva $(12,60 \mu \mathrm{mol} \mathrm{ET} / \mathrm{g})(\mathrm{Wu}$ et al. 2004), y vegetales como cebolla $(5,40 \mu \mathrm{mol}$ $\mathrm{ET} / \mathrm{g})$, brócoli $(3,36 \mu \mathrm{mol} \mathrm{ET/g})$, zanahoria $(2,12$ $\mu \mathrm{mol} \mathrm{ET/g})$ y espinaca $(7,35 \mu \mathrm{mol} \mathrm{ET} / \mathrm{g})($ Ninfali y Bacchiocca 2003). El pejibaye es superado en su capacidad antioxidante por algunas frutas, tales como arándano (Kalt et al. 1999), ciruela y fresa (Wang et al. 1996).

Los valores de grasa y almidón obtenidos son similares a los reportados por otros autores. Carrera (1998) encontró en 6 diferentes fenotipos procedentes de Perú contenidos de grasa entre $4,93 \%$ y $14,51 \%$ y entre $58,2 \%$ y $84,2 \%$ de almidón en base seca. Leterme et al. (2005) determinaron en promedio $11,4 \%$ de grasa y entre $59 \%$ y $78 \%$ de almidón en base seca para 17 genotipos procedentes de Colombia. Mientras que los valores obtenidos de carotenoides son superiores a los reportados por Rodriguez-Amaya (1996) quien obtuvo 43,2 $\mu \mathrm{g} / \mathrm{g}$ de carotenoides en base seca con pulpa cocida de pejibayes procedentes de Brasil. De Rosso y Mercadante (2007) obtuvieron $197,7 \mu \mathrm{g} / \mathrm{g}$ en base seca con pulpa cocida, pero por medio del método HPLC-PDA-MS/MS, distinto al método espectrofotométrico utilizado en este trabajo.

Se encontraron diferencias significativas $(\mathrm{p}<0,1)$ para el contenido de grasa y la capacidad antioxidante. Se obtuvo un mayor contenido de grasa conforme mayor fue el índice de cosecha, lo que indica que la planta acumula grasa en los frutos conforme aumenta la madurez del racimo. Por su parte, la capacidad antioxidante tuvo valores mayores entre menor fue el índice de cosecha, aunque esto podría deberse al método utilizado.

No se encontraron diferencias significativas entre las edades de cosecha para el contenido de almidón y el contenido de carotenoides. Posiblemente por la variabilidad genética del pejibaye se requiera un mayor tamaño de muestra en estas variables para declarar diferencias estadísticas. Se presume esto con base en los resultados obtenidos en color externo, color de la pulpa y firmeza, ya que en estas variables sí se encontraron diferencias significativas cuando se utilizó una muestra considerablemente mayor. 
En conjunto los resultados de este estudio permiten concluir que el pejibaye es un fruto que se podría clasificar como no climatérico, sin embargo queda por investigar, la producción de etileno en poscosecha. Presenta una tasa respiratoria alta que a su vez le confiere una vida útil poscosecha corta; se determinó un bajo porcentaje de acidez que eventualmente podría favorecer el desarrollo de microorganismos que acortan aún más su vida útil. Variables como la firmeza de la pulpa, el color de pulpa, el color de cáscara, el contenido de sólidos solubles, el contenido de grasa y la capacidad antioxidante dependen de la edad de cosecha. Cosechar los frutos con mayor índice de cosecha permite obtener frutos menos firmes, con un mayor contenido de grasa y almidones, así como también con una pulpa y cáscara más roja. Aunque el color externo y el contenido de sólidos solubles pueden experimentar cambios durante el almacenamiento, estos cambios no tienen la magnitud que experimentan los frutos climatéricos (Kays 1997).

Además de su ya conocido valor nutritivo, se determinó que el pejibaye tiene una alta capacidad antioxidante, superior a la que presentan otras frutas, la cual es mayor en el índice de cosecha más temprano. A pesar de estos resultados, no sería recomendable la cosecha temprana para obtener una mayor capacidad antioxidante. Esto porque los carotenoides son un importante componente nutricional que aporta esta fruta (Mora-Urpí et al. 1997), los cuales están presentes en mayor cantidad en aquellas frutas con epicarpo y pulpa roja (Johannessen 1966), y en esta investigación se determinó que las frutas cosechadas con mayor índice de cosecha tenían un epicarpo y una pulpa más roja. También debe considerarse que el método ORAC mide con facilidad la acción de compuestos hidrofílicos como la vitamina $\mathrm{C}$ y los polifenoles, pero no así la actividad antioxidante de los carotenoides (Huang et al. 2000), por lo cual la capacidad antioxidante total se podría subestimar, debido a que en la determinación con el método ORAC no se considera el aporte de los carotenoides presentes en los pejibayes evaluados.

\section{AGRADECIMIENTOS}

Se agradece al Dr. Jorge Mora-Urpí y a los funcionarios de la Estación Experimental Los Diamantes por la colaboración brindada para la realización de esta investigación. También se agradece al laboratorio de química del CITA por la colaboración brindada para realizar los análisis químicos aquí presentados. Esta investigación fue financiada por el proyecto PAVUC (Producing Added Value from Under-utilised tropical fruit Crops), PAVUC-FP6-INCO project-DEV 2, contract 015279 .

\section{LITERATURA CITADA}

AOAC (OFFICIAL METHODS OF ANALYSIS). 1990. 15ta ed. Arlington, VA, USA: Association of Official Analytical Chemists.

BARKAI-GOLAN R. 2001. Postharvest diseases of fruits and vegetables: development and control. London, Elsevier. $418 \mathrm{p}$

BOWER J.H., BIASI W.V., MITCHAM E.J. 2003. Effects of ethylene and 1-MCP on the quality and storage life of strawberries. Postharvest Biology and Technology 28:417-423

BRITTON G., LIAAEN-JENSEN S., PFANDER H. 1995. Carotenoids: Isolation and analysis, Birkhauser Verlag, Alemania. p. 95, 102-107.

CARRERA L. 1999. Isolation and characterisation of pejibaye starch. Journal of Applied Botany 73:122 127.

CHAVES O. 2005. Factores involucrados en el deterioro poscosecha de pejibaye y evaluación de tratamientos para prolongar su vida útil. Tesis de licenciatura en Agronomía con énfasis en Fitotecnia. Liberia, Universidad de Costa Rica, Sede de Guanacaste. $82 \mathrm{p}$.

CITA. 2007. Determinación de carotenoides. Método de análisis AQCITA-M035. San José, Costa Rica, Centro Nacional de Ciencia y Tecnología de Alimentos (CITA). 5 p.

DE ROSSO V.V., MERCADANTE A.Z. 2007. Identification and quantification of carotenoids, by HPLCPDA-MS/MS, from Amazonian fruits. Journal of Agricultural and Food Chemistry 55:5062-5072. 
FERNÁNDEZ M. 1988. Definición de las características químico-nutricionales de cuatro poblaciones de pejibaye (Bactris gasipaes H.B.K.). Tesis de licenciatura en Tecnología de Alimentos. San José, Costa Rica, Universidad de Costa Rica. 54 p.

GARAICO K.C., ESPINOZA P.Y. 1993. Efectos del almacenamiento en frío en la conservación del chontaduro (Bactris gasipaes Kunth). In Congreso internacional sobre biología, agronomía e industrialización del pijuayo (IV, 1991, Iquitos, Perú). Memorias. Eds. J, Mora Urpí; LT, Szott; M, Murillo; VM, Patiño. San José, CR, Editorial de la Universidad de Costa Rica. 492 p.

GIOVANNONI J. 2001. Molecular biology of fruit maturation and ripening. Annual Review of Plant Physiology and Plant Molecular Biology 52:725-749.

GOLDSCHMIDT E.E. 1997. Ripening of citrus and other non-climacteric fruits: a role for ethylene. Acta Hort. 463:335-340.

GÓMEZ G. 1990. Desarrollo y evaluación de un alimento infantil con base en pejibaye (Bactris gasipaes). Tesis de licenciatura en Nutrición. San José, Costa Rica, Universidad de Costa Rica. 81 p.

HARDENBURG R.E., WATADA A.E., WANG C.Y. 1998. Almacenamiento comercial de frutas, legumbres y existencias de floristerías y viveros. San José, CR: IICA. $150 \mathrm{p}$.

HERNÁNDEZ J.A., MORA-URPÍ J., ROCHA O. 2008. Diversidad genética y relaciones de parentesco de las poblaciones silvestres y cultivadas de pejibaye (Bactris gasipaes, Palmae), utilizando marcadores microsatelitales. 2008. Rev. Biol. Trop 56(1):217-245.

HOLM J., BJORCK I., DREWS A., ASP N.G. 1986. A rapid method for the analysis of starch. Starch/Starke 38:224-229.

JOHANNESSEN C.L. 1967. Pejibaye palm: physical and chemical analysis of the fruit. Economic Botany 21(4):371-377.

KALT W., FOMEY C.F., MARTIN A., PRIOR R.L. 1999. Antioxidant Capacity, Vitamin C, Phenolics, and Anthocyanins after Fresh Storage of Small Fruits. Journal of Agricultural and Food Chemistry 47:4638-4644.

KAYS S. 1997. Postharvest physiology of perishable plant products. Athens, Georgia, US, Exon Press. 531 p.
LEÓN J. 1987. Botánica de los cultivos tropicales. San José, CR, IICA. p. 60.

LETERME P., GARCÍA MF., LONDON, AM., ROJAS MG., BULDGEN A., SOUFFRANT WB. 2005. Chemical composition and nutritive value of peach palm (Bactris gasipaes Kunth) in rats. Journal of the Science of Food and Agriculture 85:1505-1512.

MATILE P., HORTENSTEINER S., THOMAS H. 1999. Chlorophyll degradation. Annual Review of Plant Physiology and Plant Molecular Biology. 50:67-95.

MENCACA D., MURILLO E. 2006. Fraccionamiento y caracterización de los principales carotenoides del zapote (Quararibea cordata). Panamá, Universidad de Panamá. p. 2-12.

MORA-URPÍ J. 1983. El pejibaye (Bactris gasipaes H.B.K.): origen, biología floral y manejo agronómico. In Palmeras poco utilizadas en América Tropical. Turrialba, Costa Rica, CATIE. p. 118-160.

MORA-URPÍ J., BOGANTES A., ARROYO C. 1999 Cultivares de pejibaye para palmito, pp. 41-47. In: J. Mora-Urpí y Javier Gainza (eds.). Palmito de pejibaye. Editorial Universidad de Costa Rica.

MORA-URPÍ J., WEBER J.C., CLEMENT C.R. 1997. Peach Palm (Bactris gasipaes Kunth): Promoting the conservation and use of underutilized and neglected crops. Rome, IT, Institute of Plant Genetics and Crop Plant Research. 83 p.

NINFALI P., BACCHIOCCA A.M. 2003. Polyphenols and antioxidant capacity of vegetables under fresh and frozen conditions. Journal of Agricultural and Food Chemistry 51:2222-2226.

O’HARE T.J. 1997a. Carambola. In Mitra, S. ed. Postharvest physiology and storage of tropical and subtropical fruits. UK, CAB International. p. 295-307.

O’HARE T.J. 1997b. Rambutan. In Mitra, S. ed. Postharvest physiology and storage of tropical and subtropical fruits. UK, CAB International. p. 309-321.

OU B., HAMPSCH-WOODILL M., PRIOR R.L. 2001 Development and validation of an improved oxygen radical absorbance capacity assay using fluorescein as the fluorescent probe. Journal of Agricultural and Food Chemistry 49:4619-4626.

RODRIGUEZ-AMAYA D.B. 1996. Assessment of the provitamin A contents of foods: the Brazilian experience. Journal of Food Composition and Analysis (9):196-230. 
RODRÍGUEZ-AMAYA D.B. 1997. Carotenoids and food preparation: The retention of provitamin A carotenoids in prepared, processed, and stored foods. Campinas, Brazil, Departamento de Ciencias de Alimentos, Universidad Estatal de Campinas. 93 p.

RODRÍGUEZ-AMAYA D.B. 1999. A guide to carotenoid analysis in foods. Brasil Universidade Estadual de Capinas. p. 34-45.

SÁENZ M.V., VALVERDE E., VARGAS A. 1992. Cosecha y empaque de pejibaye como fruta fresca. Boletín Pejibaye IV (1):2-5.

SAURA-CALIXTO F., GOÑI I., BRAVO L., MAÑAS E. 1993. Resistant starch in foods. Modified method for dietary fiber residues. Journal of Food Science 58: 642-643.
SCHOUTEN R.E., ZHANG X., VERSCHOR J.A., OTMA E.C., TIJSKENS L.M., VAN KOOTEN O. 2009. Development of colour of broccoli heads as affected by controlled atmosphere storage and temperature. Postharvest Biology and Technology 51:27-35.

SEYMOUR G.B., TAYLOR J.E., TUCKER G.A. 1993. Biochemistry of fruit ripening. London, Chapman and Hall. 454 p.

WANG H., CAO G., PRIOR R.L. 1996. Total antioxidant capacity of fruits. Journal of Agricultural and Food Chemistry 44:701-705.

WU X., BEECHER G.R., HOLDEN J.M., HAYTOWITZ D.B., GEBHARDT S.E., PRIOR R.L. 2004. Lipophilic and hydrophilic antioxidant capacities of common foods in the United States. Journal of Agricultural and Food Chemistry 52:4026-4037. 
